\title{
Effect of Electric Field and Temperature on Average Structure and Domain Wall Motion in $0.93 \mathrm{Bi}_{0.5} \mathrm{Na}_{0.5} \mathrm{TiO}_{3}-\mathrm{-0.07}_{\mathrm{BaTiO}} \mathrm{Ceramic}_{3}$
}

\author{
Jian Wang, ${ }^{1}$ Yun Liu, ${ }^{1}$ Andrew Studer, ${ }^{2}$ Lasse Norén, ${ }^{1}$ and Ray Withers ${ }^{1}$ \\ ${ }^{1}$ Research School of Chemistry, The Australian National University, Canberra, ACT 0200, Australia \\ ${ }^{2}$ Bragg Institute, ANSTO, Lucas Heights, NSW 2232, Australia \\ Correspondence should be addressed to Yun Liu; yliu@rsc.anu.edu.au
}

Received 3 June 2013; Accepted 24 June 2013

Academic Editor: Danyang Wang

Copyright ( 2013 Jian Wang et al. This is an open access article distributed under the Creative Commons Attribution License, which permits unrestricted use, distribution, and reproduction in any medium, provided the original work is properly cited.

In situ neutron powder diffraction patterns and dielectric spectra of $0.93 \mathrm{Bi}_{0.5} \mathrm{Na}_{0.5} \mathrm{TiO}_{3}-0.07 \mathrm{BaTiO}_{3}$ ceramic were investigated under different electrical fields and temperatures. An electric-field-induced phase transition from metrically cubic to metrically tetragonal, associated with strong domain wall motion, occurs. Such induced phase and domain wall motion are unchanged until the hightemperature phase transition occurs from metrically tetragonal to metrically cubic. All these changes are irrelevant to the observed depolarization temperature $\left(75^{\circ} \mathrm{C}\right)$. The depolarization behaviour is thus suggested to be associated with the local structure caused by the octahedral tilt twinning disorder.

\section{Introduction}

Piezoelectric materials are used in a variety of devices such as stress sensors, ultrasound emitters, and large-strain actuators. The ultrahigh strain $(\sim 1.7 \%)$ in $\langle 001\rangle$ oriented rhombohedral $\mathrm{Pb}\left(\mathrm{Zn}_{1 / 3} \mathrm{Nb}_{2 / 3}\right) \mathrm{O}_{3}-\mathrm{PbTiO}_{3}$ and $\mathrm{Pb}\left(\mathrm{Mg}_{1 / 3} \mathrm{Nb}_{2 / 3}\right) \mathrm{O}_{3}-\mathrm{PbTiO}_{3}$ single crystals is suggested to be a result of the electricfield-induced rhombohedral-tetragonal phase transition [14]. Lead-free $\mathrm{BiFeO}_{3}$ films have a reversible electric-fieldinduced strain of over $5 \%$ that arises from moving the boundaries between tetragonal- and rhombohedral-like phases [5]. In fact, such electric-field-induced structural transition is found to be universal in rare-earth-substituted $\mathrm{BiFeO}_{3}$ [6], resulting from isostructural transitions, disappearance and reappearance of the tilting of the oxygen octahedral, and reentrance into specific crystallographic classes [7]. This phenomenon also occurs in other lead-free piezoelectric material systems $[8,9]$.

$(1-x) \mathrm{Bi}_{0.5} \mathrm{Na}_{0.5} \mathrm{TiO}_{3}-x \mathrm{BaTiO}_{3}$ (BNTBT) ceramic has been intensively studied as a candidate for lead-free piezoelectric materials due to the high strain that appeared in the morphotropic phase boundary compositions $(0.06 \leq$ $x \leq 0.08)$ [10]. Such high strain is suggested to be related to the electric-field-induced structural changes [11-13]. It is found that BNTBT undergo a ferroelectric to antiferroelectric transition above a so-called depolarization temperature $\left(T_{d}\right)$ above which the piezoelectric properties deteriorate [14]. In this paper, we aim to investigate the variation in the structure and domain texture of $0.93 \mathrm{Bi}_{0.5} \mathrm{Na}_{0.5} \mathrm{TiO}_{3}-0.07 \mathrm{BaTiO}_{3}(x=$ 0.07 , abbreviated as BNTBT7 hereafter) ceramic under the electrical field and with increasing temperature via in situ neutron diffraction technique in order to understand the relationship between the structural change under the applied electric field and the depolarisation behaviour at elevated temperature.

\section{Experimental}

BNTBT7 ceramic was synthesized by a solid state reaction. High purity $\mathrm{Na}_{2} \mathrm{CO}_{3}, \mathrm{Bi}_{2} \mathrm{O}_{3}, \mathrm{BaCO}_{3}$, and $\mathrm{TiO}_{2}$ were used as starting materials. Mixtures of the starting materials were first ball-milled for $5 \mathrm{~h}$. The dried mixture of powder was then calcined at $800^{\circ} \mathrm{C}$ for $3 \mathrm{~h}$, followed by ball-milling again for $2 \mathrm{~h}$. The resultant powders were then pressed into pellets under a uniaxial pressure of $200 \mathrm{MPa}$, using poly(vinyl 


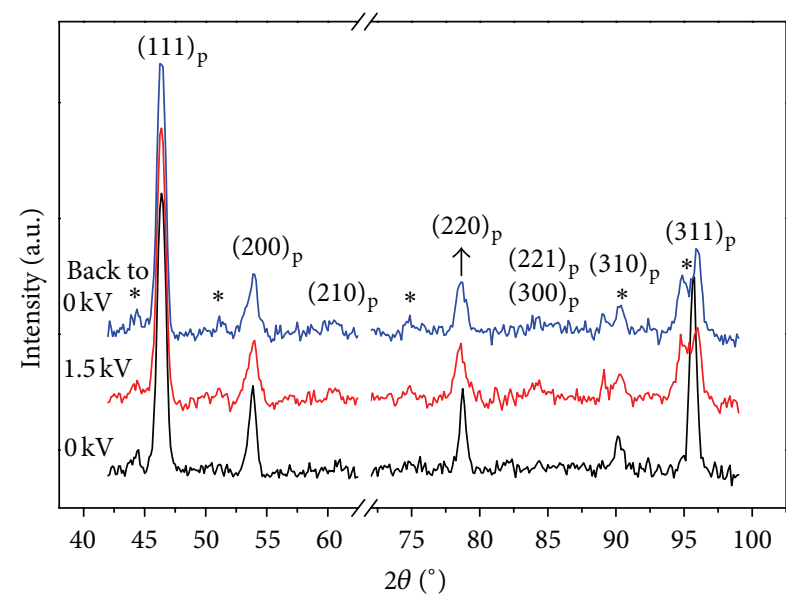

* Ag

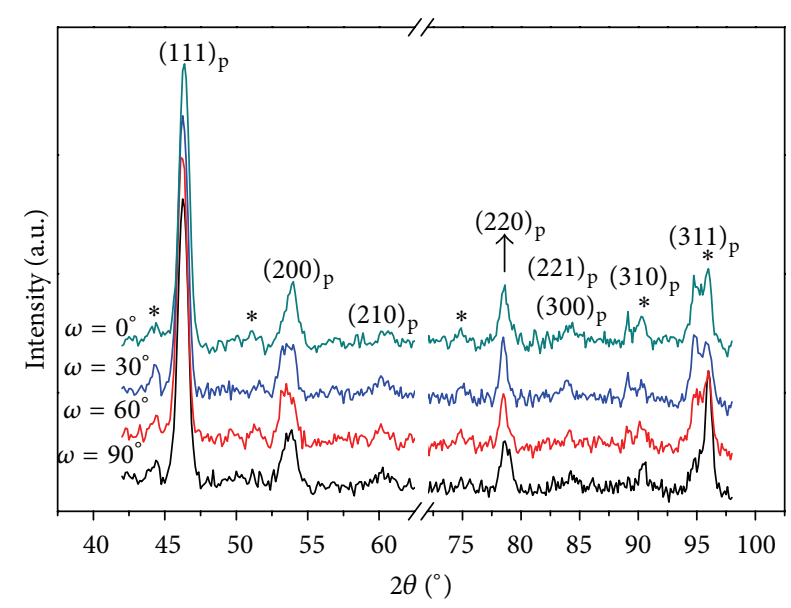

* Ag

(a)

(b)

FIGURE 1: Neutron diffraction patterns of BNTBT7 collected at room temperature (a) under different applied electric fields and at $\omega=0^{\circ}$ and (b) under $1.5 \mathrm{kV}$ and at different $\omega$ angles.

alcohol) as a binder. Finally, the as-sintered ceramics were polished on both sides down to $0.8 \mathrm{~mm}$ thickness and coated with silver paste, followed by the heat treatment at $500^{\circ} \mathrm{C}$ for $5 \mathrm{~min}$. The dielectric properties of the sample were measured using a high precision LCR meter (HP 4284A).

In situ neutron powder diffraction measurements were carried out as a function of applied electric field and temperature. These measurements were completed using the WOMBAT instrument on the OPAL reactor at the Australian Nuclear Science and Technology Organisation (ANSTO). A Ge (115) monochromator was used to select a wavelength of $\lambda=1.727 \AA$. The sample was fixed perpendicular to a central stage on WOMBAT. Conducting wires were attached to the silver coating on either side of the sample to enable a voltage to be applied. The sample was rotated with the cylindrical axis of the stage to vary the angle $(\omega)$ between the incident beam and the poling direction from $90^{\circ}$ to $0^{\circ}$ in $30^{\circ}$ increments.

\section{Results and Discussion}

Figure 1(a) shows the room temperature diffraction pattern of BNTBT7 ceramic collected under different electric fields and at $\omega=0^{\circ}$. There is no apparent peak splitting in BNTBT7 prior to the application of electric field, suggesting that its average structure is metrically cubic. Once the electric field is applied (e.g., $1.5 \mathrm{kV}$ ), we can see a clear peak splitting in $(200)_{p},(220)_{p}$, and $(311)_{p}$ diffraction peaks, but not in $(111)_{\mathrm{p}}$ peak. This demonstrates a clear phase transition from metrically cubic to metrically tetragonal. Note that both (113) and $(311)_{p}$ peak intensities are almost identical. After, withdrawal of the electric field, their intensities are different, even though the peak splitting profile is retained. This suggests that the metrically tetragonal phase would be partially reversed after the withdrawal of the electric field. Figure 1(b) is the diffraction patterns under an applied electric field of $1.5 \mathrm{kV}$ as a function of $\omega$. There is a clear splitting of $(200)_{p},(220)_{p}$, and $(311)_{\mathrm{p}}$ peaks once $\omega$ changes, where the relative intensity of these splitting peaks is different. The results are in accordance with Daniels' work [11]. The applied electric field causes non$180^{\circ}$ domain wall motion, which can be detected using in situ neutron diffraction or X-ray diffraction as a consequence of the variation in the relative intensities of some diffraction peaks. For example, a $90^{\circ}$ domain switching in tetragonal phase is demonstrated through the relative intensity change, for example, between the (002) and $(200) /(020)$ peaks, or between the $(202) /(022)$ and (220) peaks [15]. It is apparent that the relative intensity of the peak and its shoulder within the $(220)_{\mathrm{p}}$ varies significantly in different situations (at $0 \mathrm{KV}$ and $1.5 \mathrm{KV}$, and back to $0 \mathrm{KV}$ ), indicating that the $90^{\circ}$ domain wall motion appears under an electric field and leading to the domain texture-related preferred orientation in the structure as shown in Figure 1(b).

Figure 2(a) shows the dielectric constant and loss tangent of poled BNTBT7 ceramics as a function of temperature. A small jump in the dielectric constant and loss tangent is observed at about $70^{\circ} \mathrm{C}$ which is regarded as the depolarization temperature $T_{d}$ for the sample. Figure 2(b) shows in situ neutron powder diffraction patterns collected at different temperatures up to $200^{\circ} \mathrm{C}$ at $\omega=0^{\circ}$. Note that a dc bias of $0.5 \mathrm{kV}$ is applied during the measurement to enhance the depolarization effect. The intensity and the profile of the diffraction peaks below $100^{\circ} \mathrm{C}$ are almost the same. The splitting of $(310)_{\mathrm{p}}$ and $(311)_{\mathrm{p}}$ peaks as well as the shoulders of the $(200)_{p}$ and $(220)_{p}$ peaks disappears at $150^{\circ} \mathrm{C}$. This indicates that a transition from metrically tetragonal to metrically cubic occurs in the vicinity of this temperature. This phase transition narrows the dielectric polarisation relaxation in the corresponding temperature range (see Figure 2(a)). Figure 2(c) shows the diffraction patterns of the $(311)_{p}$ peak at different temperatures (room temperature, $75^{\circ} \mathrm{C}$, and $100^{\circ} \mathrm{C}$ ) and different $\omega$ angles in order to investigate the structural evolution at $T_{d}$. For all selected temperatures, the intensity 


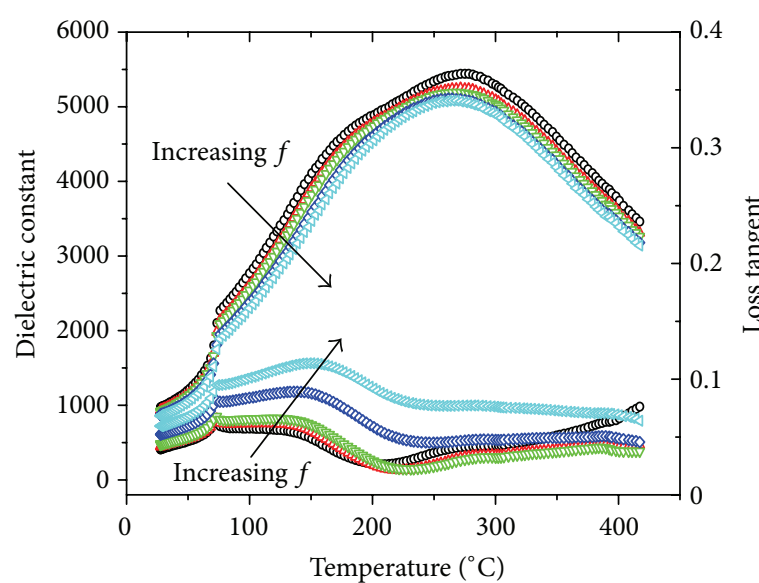

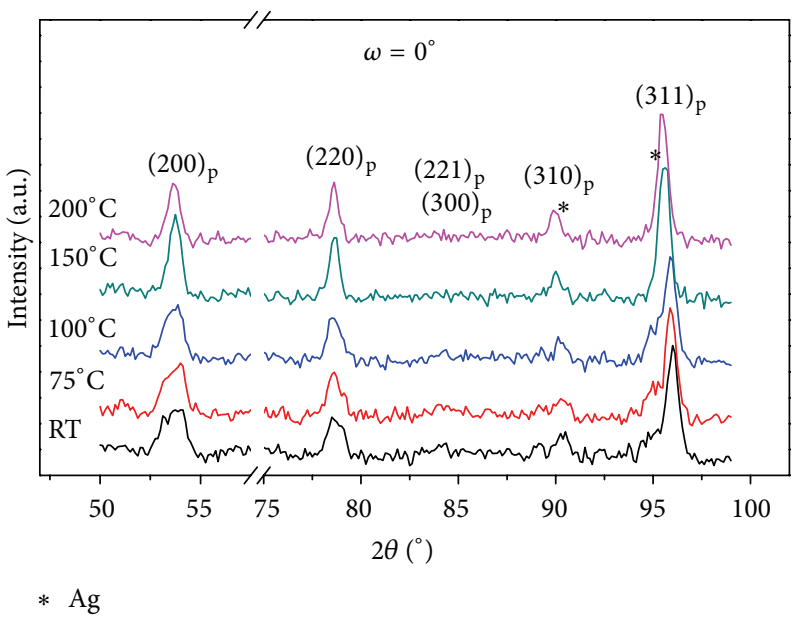

(b)
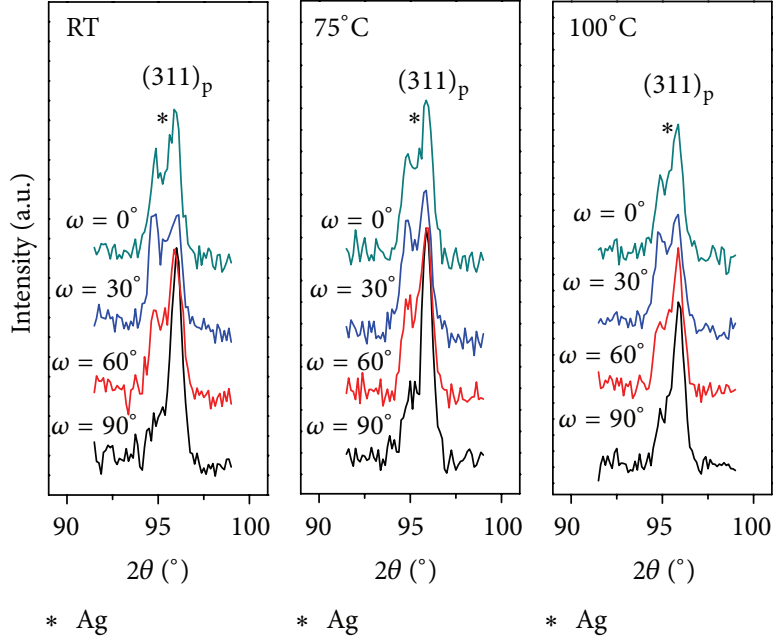

(c)

Figure 2: (a) Temperature dependence on dielectric spectra of BNTBT7 ceramic and (b) its neutron diffraction patterns at $\omega=0^{\circ}$ as well as (c) a typical (311) $)_{\mathrm{p}}$ diffraction peak at different temperatures and different $\omega$ angles.

of the induced (113) peak (left hand side one) increases with respect to the $\omega$ angle and reaches the highest value relative to the (311) peak (right hand side one) at $\omega=60^{\circ}$. There is no change in the preferred domain orientation with increasing temperature that can be revealed by neutron diffraction, indicating that the depolarization behaviour is not determined by the domain wall motion. Thus, we conclude that both the average structure and domain wall motion follow the same trend as the temperature increases, and there is no observable difference below and above $T_{d}$.

In BNTBT ceramics, the octahedral tilt twin disordering and the resultant orthorhombic Pnma interfacial phase in the material matrix have been reported [14]. As a result, an increasing volume of such local structure with respect to the electrical field results in an observable metrically tetragonal phase. The $T_{d}$ is irrelevant to or at least not dominated by the average structure and domain wall motion. Taking into consideration its local structure, it is highly possible that the $T_{d}$ is the balancing sequence of the following possible co-existing factors: an incomplete poling and/or unpoled parts within the ceramic, which still keep the original metrically cubic phase; the octahedral tilt twinning disorder; and high temperature metrically cubic phase induced with the increasing temperature.

\section{Conclusions}

An electric-field-induced phase transition from metrically cubic to metrically tetragonal, which is associated with a strong $90^{\circ}$ domain wall motion, is observed in BNTBT7 ceramic. The temperature-dependent diffraction patterns show that a high-temperature phase transition from induced metrically tetragonal to metrically cubic occurs between $100^{\circ} \mathrm{C}$ and $150^{\circ} \mathrm{C}$, as the temperature increases. However, neither the average structure nor the domain wall motion induced orientation changes are relevant to the depolarization temperature. 


\section{Acknowledgments}

Jian Wang, Yun Liu, and Ray Withers acknowledge the financial support from the Australian Research Council (ARC) in the form of an ARC Discovery Grant. Yun Liu also acknowledges support from the ARC Future Fellowships program. Jian Wang, Yun Liu, and Andrew Studer also thank the Australian Institute of Nuclear Science and Engineering (ANSIE) for the financial support to access the national neutron facilities at ANSTO.

\section{References}

[1] S.-E. Park and T. R. Shrout, "Ultrahigh strain and piezoelectric behavior in relaxor based ferroelectric single crystals," Journal of Applied Physics, vol. 82, no. 4, pp. 1804-1811, 1997.

[2] M. Davis, D. Damjanovic, and N. Setter, "Electric-field-, temperature-, and stress-induced phase transitions in relaxor ferroelectric single crystals," Physical Review B, vol. 73, no. 1, Article ID 014115, 2006.

[3] H. Y. Chen, C. S. Tu, C. M. Hung et al., "Poling effect and piezoelectric response in high-strain ferroelectric $0.70 \mathrm{~Pb}$ $\left(\mathrm{Mg}_{1 / 3} \mathrm{Nb}_{2 / 3}\right) \mathrm{O}_{3}-0.30 \quad \mathrm{PbTiO}_{3}$ crystal," Journal of Applied Physics, vol. 108, no. 4, Article ID 044101, 2010.

[4] C. Zhan, J. Wu, S. Yin, and X. Jiang, "Strong, high-frequency, ac electric-field-induced rhombohedra-tetragonal phase transition in $\mathrm{Pb}\left(\mathrm{Mg}_{1 / 3} \mathrm{Nb}_{2 / 3}\right) \mathrm{O}_{3}-\mathrm{PbTiO}_{3}$ single crystal," Journal of Applied Physics, vol. 97, no. 7, Article ID 074107, pp. 1-4, 2005.

[5] J. X. Zhang, B. Xiang, Q. He et al., "Large field-induced strains in a lead-free piezoelectric material," Nature Nanotechnology, vol. 6, no. 2, pp. 98-102, 2011.

[6] D. Kan, L. Pálová, V. Anbusathaiah et al., "Universal behavior and electric-field-lnduced structural transition in rare-earthsubstituted $\mathrm{BiFeO}_{3}$," Advanced Functional Materials, vol. 20, no. 7, pp. 1108-1115, 2010.

[7] S. Lisenkov, D. Rahmedov, and L. Bellaiche, "Electric-fieldinduced paths in multiferroic $\mathrm{BiFeO}_{3}$ from atomistic simulations," Physical Review Letters, vol. 103, no. 4, Article ID 047204, 2009.

[8] A. J. Royles, A. J. Bell, J. E. Daniels, S. J. Milne, and T. P. Comyn, "Observation of a time-dependent structural phase transition in potassium sodium bismuth titanate," Applied Physics Letters, vol. 98, no. 18, Article ID 182904, 2011.

[9] M. Hinterstein, M. Knapp, M. Hölzel et al., "Field-induced phase transition in $\mathrm{Bi}_{1 / 2} \mathrm{Na}_{1 / 2} \mathrm{TiO}_{3}$-based lead-free piezoelectric ceramics," Journal of Applied Crystallography, vol. 43, no. 6, pp. 1314-1321, 2010.

[10] T. Takenaka, K. I. Maruyama, and K. Sakata, “ $\left(\mathrm{Bi}_{1 / 2} \mathrm{Na}_{1 / 2}\right) \mathrm{TiO}_{3}$ $\mathrm{BaTiO}_{3}$ system for lead-free piezoelectric ceramics," Japanese Journal of Applied Physics, vol. 30, no. 9 B, pp. 2236-2239, 1991.

[11] J. E. Daniels, W. Jo, J. Rödel, and J. L. Jones, "Electric-fieldinduced phase transformation at a lead-free morphotropic phase boundary: case study in a $93 \%\left(\mathrm{Bi}_{0.5} \mathrm{Na}_{0.5}\right) \mathrm{TiO}_{3}$ $7 \% \mathrm{BaTiO}_{3}$ piezoelectric ceramic," Applied Physics Letters, vol. 95, no. 3, Article ID 032904, 2009.

[12] H. Simons, J. Daniels, W. Jo et al., "Electric-field-induced strain mechanisms in lead-free $94 \%\left(\mathrm{Bi}_{1 / 2} \mathrm{Na}_{1 / 2}\right) \mathrm{TiO}_{3}-6 \% \mathrm{BaTiO}_{3}$," Applied Physics Letters, vol. 98, no. 8, Article ID 082901, 2011.

[13] C. Ma, X. Tan, E. Dul'Kin, and M. Roth, "Domain structure-dielectric property relationship in lead-free
$(1-x)\left(\mathrm{Bi}_{1 / 2} \mathrm{Na}_{1 / 2}\right) \mathrm{TiO}_{3}-x \mathrm{BaTiO}_{3}$ ceramics," Journal of Applied Physics, vol. 108, no. 10, Article ID 104105, 2010.

[14] Y. Guo, Y. Liu, R. L. Withers, F. Brink, and H. Chen, "Large electric field-induced strain and antiferroelectric behavior in (1$x)\left(\mathrm{Na}_{0.5} \mathrm{Bi}_{0.5}\right) \mathrm{TiO}_{3}-x \mathrm{BaTiO}_{3}$ ceramics," Chemistry of Materials, vol. 23, no. 2, pp. 219-228, 2011.

[15] J. L. Jones, "The use of diffraction in the characterization of piezoelectric materials," Journal of Electroceramics, vol. 19, no. 1, pp. 67-79, 2007. 

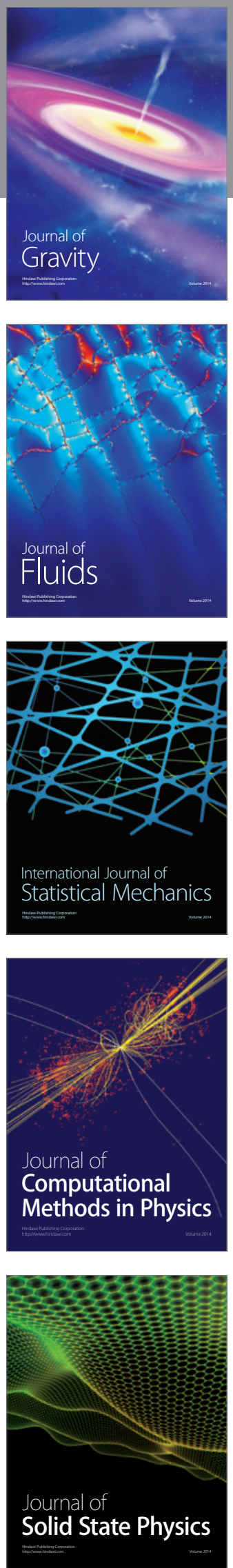

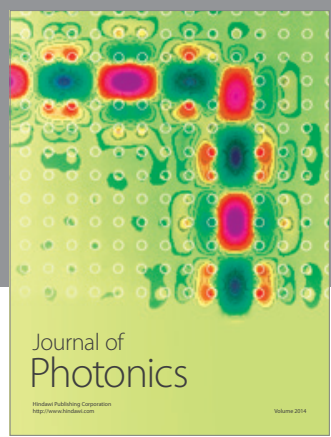

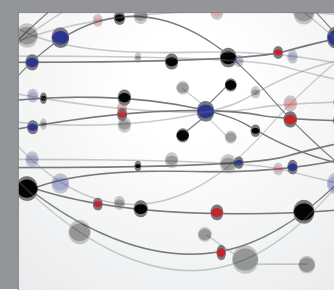

The Scientific World Journal

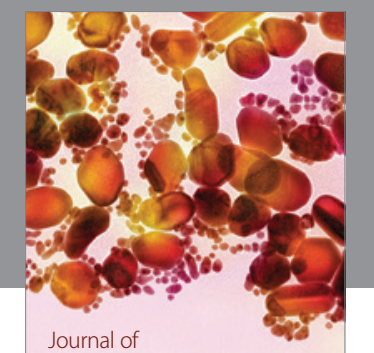

Soft Matter
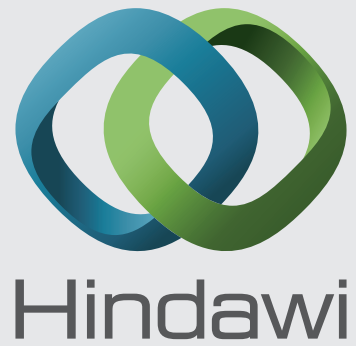

Submit your manuscripts at

http://www.hindawi.com
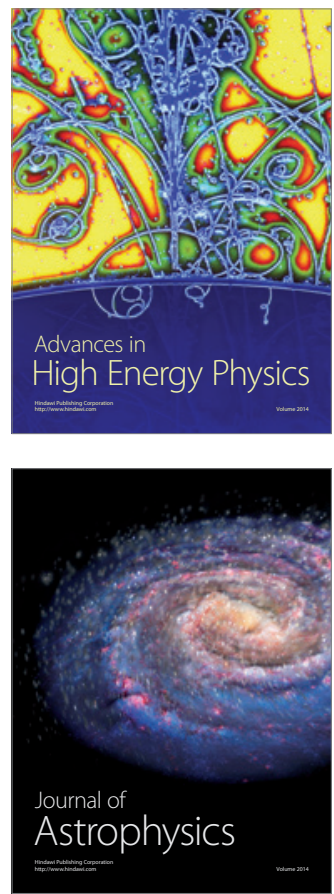
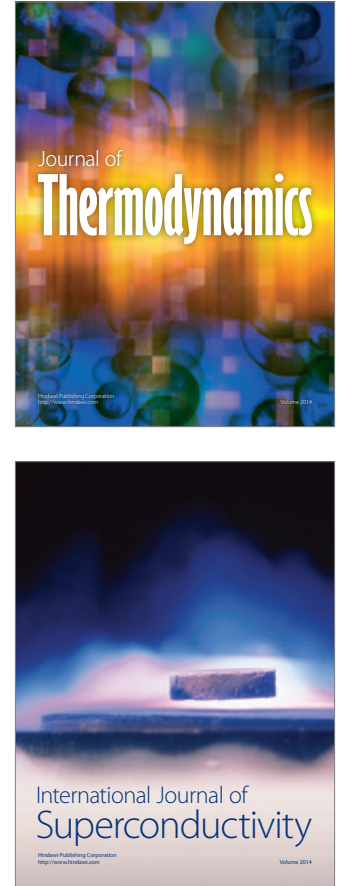
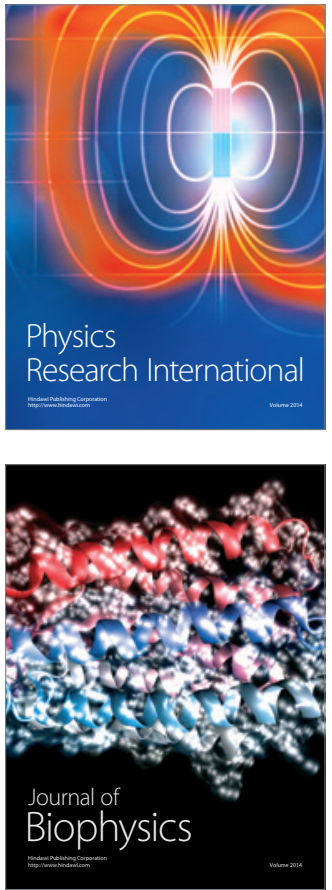
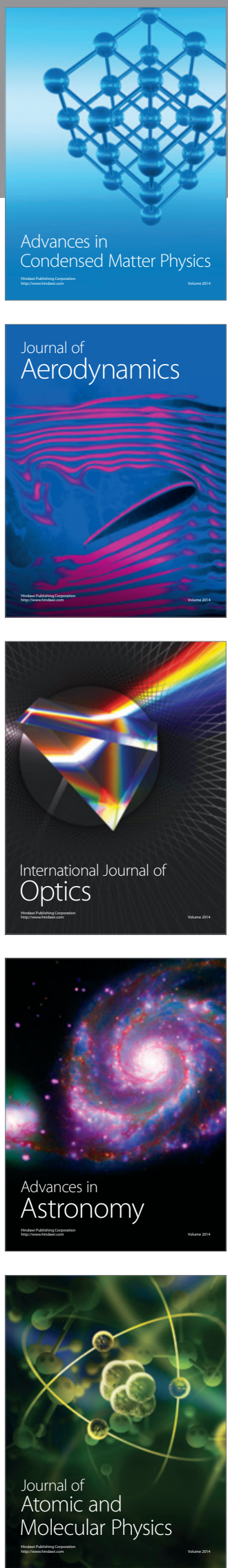